\title{
Observations of the coronal dynamics associated with solar radio spike burst emission $\star$
}

\author{
J. I. Khan ${ }^{1}$ and H. Aurass ${ }^{2}$ \\ 1 Department of Physics and Astronomy, University of Glasgow, Glasgow G12 8QQ, Scotland, UK \\ e-mail: jkhan@spd.aas.org \\ 2 Astrophysikalisches Institut Potsdam, An der Sternwarte 16, 14482 Potsdam, Germany \\ Received 11 August 2005 / Accepted 2 June 2006
}

ABSTRACT

\begin{abstract}
We present the results of an analysis of multi-wavelength data for a solar radio spike burst event which was observed on 1997 November 14 by the Solar Radio Spectrometers of the Astrophysikalisches Institut Potsdam (AIP). Using radio imaging data from the Nançay Radioheliograph we are able to determine the location of the spike burst emission sites in relation to the associated flare and its coronal environment as seen in images from the Yohkoh Soft X-ray Telescope (SXT), Hard X-ray Telescope (HXT) and the Solar and Heliospheric Observatory (SOHO) Extreme ultraviolet Imaging Telescope (EIT). For this event we find that the spike burst emission originated high in the corona, $\sim 122 \mathrm{Mm}$ above the soft X-ray and hard X-ray emitting regions of the associated (near-limb) flaring region. Using imaging data from SXT, HXT, and EIT we are also able to show, for the first time, the location of a solar radio spike burst in relation to the dynamics of coronal structures. We find that as an earlier flare-associated coronal mass ejection (CME) in the vicinity expanded outwards it appeared to compress the magnetic field structures at its flanks (associated with highly inclined coronal loops at the periphery of the active region). The radio spike bursts appeared at the time and in the vicinity of the compression of the coronal field. The compression presumably led to reconnection of the coronal magnetic field structures, particle acceleration and the radio spike burst emission signatures. For the specific event we analyse we find that the spike burst emission is associated with secondary flare energy release induced by an earlier flare-associated CME. These observations also provide evidence for a new category of sympathetic flaring where a large flare-associated coronal mass ejection compresses adjacent magnetic structures leading to nearby, but remote, small-scale flaring.
\end{abstract}

Key words. Sun: corona - Sun: coronal mass ejections (CMEs) - Sun: flares - Sun: radio radiation - Sun: X-rays, gamma rays magnetohydrodynamics (MHD)

\section{Introduction}

Radio spike bursts (e.g., Benz 1986; Bárta \& Karlický 2001; Messmer \& Benz 2000) are a particular type of radio emission characterised by narrow bandwidth $(\Delta v / v \approx 1 \%)$, short duration $(\leq 0.1 \mathrm{~s})$, and high brightness temperature $\left(T_{\mathrm{B}} \approx 10^{15} \mathrm{~K}\right)$. The sites of this emission must have very small source volumes and they are not necessarily expected to be associated with detectable brightenings at other wavelengths.

In a dynamic radio spectrogram, radio spike bursts appear as a cloud or patch of bright points of emission which are sometimes observed during the primary energy release phase of a flare (Benz 1986). Radio spike bursts are observed in the decimetre and meter wavelength ranges, sometimes associated with other radio burst signatures (e.g., during dm continuum enhancements, near the onset of type III radio bursts, Benz 1986). Radio spike bursts are generally believed to be an emission signature of particles, at, or near to, the electron acceleration site itself.

Impulsive hard X-ray emission during flares provides another signature of accelerated particles which emanate from the acceleration site. Usually, the hard X-ray emission originates low in the corona and is believed to result from thick-target bremsstrahlung of downward propagating electrons impinging on the dense chromosphere. However elevated hard X-ray

\footnotetext{
$\star$ Movies are only available in electronic form at
} http://www.edpsciences.org emission has also been observed located at, or above, flaring soft X-ray loops (e.g., Masuda et al. 1994; Veronig \& Brown 2004). This has been attributed to emission from, e.g., (a) hot plasma located near standing fast-mode shocks created by magnetic reconnection outflow jets impacting dense coronal loops below (Tsuneta et al. 1997; Aurass \& Mann 2004; Mann et al. 2006), (b) the current sheet associated with magnetic reconnection itself or its heated outflow regions (Sui \& Holman 2003), (c) particles trapped, and possibly further accelerated, near the tops of magnetic loops formed below a reconnecting magnetic structure (Somov \& Kosugi 1997; Fletcher \& Martens 1998), or (d) thicktarget bremsstrahlung of non-thermal particles in dense coronal loops (Wheatland \& Melrose 1995; Veronig \& Brown 2004). The temporal correlation between radio spike bursts and impulsive hard X-ray bursts is often close, with the fluxes showing, on average, similar, but not identical behaviour (e.g., Aschwanden et al. 1985; Aschwanden \& Güdel 1992). Consequently, detailed comparisons of radio spike burst source sites with hard X-ray images are expected to be particularly important in providing information about the specific mechanism of energy release, the process of particle acceleration, and the mechanism of radio spike burst generation.

The source locations of radio spike bursts have already been determined and examined in a few cases (e.g., Heyvaerts et al. 1978; Krucker et al. 1995, 1997; Paesold et al. 2001; Benz et al. 2002). However adequately detailed comparisons with coronal 
structures and hard X-ray sources have not yet been reported. According to our estimation this is partly due to the source positions of the particular events examined in the past, and partly due to the difficulty of detailed spike burst observations. Generally speaking, the events examined to date have been events which occurred on the solar disk. Moreover, none of these events had soft X-ray or EUV data of high temporal and spatial resolution. The comparison with coronal features for those events mostly consisted of one or two snapshots which provided merely contextual information. However, a major difficulty in this line of work is the sparsity of clearly identified radio spike burst events and the fact that the relatively narrow spike cloud bandwidth frequently does not overlap the discrete and comparatively coarsely spaced frequencies of radio imaging instruments.

In this paper we present the results of a multi-wavelength analysis of a radio spike burst event associated with a flare near the limb of the Sun. Among our various findings, we report the first observations of the coronal dynamics associated with radio spike burst emission. We find that the radio spike burst emission originates high in the corona (far from the X-ray emitting regions) near the site of the compression of magnetic loops induced by the launch of a coronal mass ejection (CME) associated with an earlier flare.

In Sect. 2 we outline the data and criteria used to select the radio spike burst event. In Sect. 3 we examine the observations for the sequence of events (before and after and) associated with the radio spike bursts. In Sect. 4 a separate and brief account is given of observations late in the sequence of events. A discussion of our results and conclusions are presented in Sect. 5. We also present movies of data associated with the spike burst event in Appendix A.

\section{Data sources and event selection}

\subsection{Data sources}

In this paper we use the following data:

- Sweep spectrograms from the Astrophysikalisches Institut Potsdam (AIP) Solar Radio Spectrometers (Mann et al. 1992). These cover the frequency range $40-800 \mathrm{MHz}$ with a sampling rate of $0.1 \mathrm{~s}$. They are used here to identify, select and analyse spike burst events.

- Whole-Sun soft X-ray fluxes from detectors on board the Geostationary Operational Environmental Satellite (GOES). These cover the wavelength ranges $\sim 0.5-4 \AA$ and $\sim 1-8 \AA$ and are used to examine the soft X-ray flares associated with spike burst events and as a time-line to show the context in which they occurred.

- Radio images taken at 327.0 MHz and 410.5 MHz from the Nançay Radioheliograph (NRH) (Kerdraon \& Delouis 1997). These are used to image the location and development of the spike burst emission sites.

- Hard X-ray Telescope (HXT) (Kosugi et al. 1991) data from the Yohkoh satellite (Ogawara et al. 1991). These are used to investigate the temporal correlation between hard X-ray and radio fluxes and to compare the sites of hard $\mathrm{X}$-ray and radio spike burst emission.

- Images from the Yohkoh Soft X-ray Telescope (SXT) (Tsuneta et al. 1991). Here we consider whole-Sun fullframe images (FFIs) as well as quiet-mode and flare-mode partial-frame images (PFIs). These are used to examine the development of coronal structures hotter than $\sim 2 \mathrm{MK}$ associated with spike burst emission.

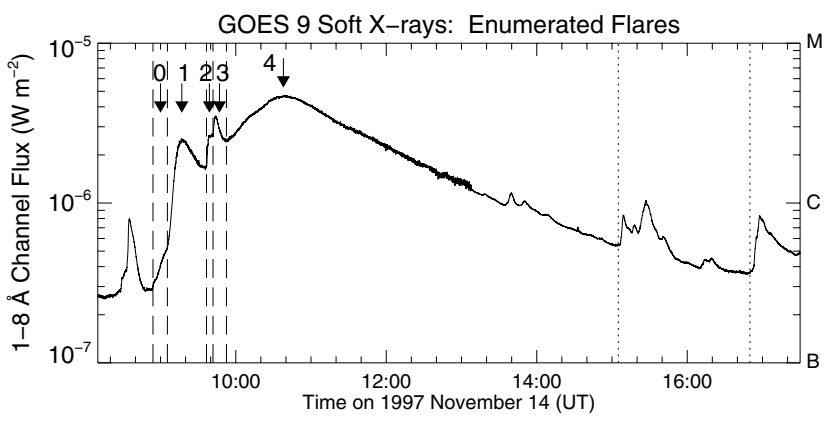

Fig. 1. The soft X-ray flux from the low energy channel ( $\sim 1-8 \AA$ ) from GOES 9 showing (enumerated) flares observed near the time of radio spike burst emission observed on 1997 November 14. This shows the overall context of the series of overlapping flares, Flares $0-4$, possibly related, directly or indirectly to the radio spike bursts.

- EUV $195 \AA$ images from the Extreme ultraviolet Imaging Telescope (EIT) (Delaboudinière et al. 1995) on board the Solar and Heliospheric Observatory (SOHO) (Domingo et al. 1995). These are used to investigate the evolution of coronal structures at $\sim 1.6 \mathrm{MK}$ associated with spike burst emission.

\subsection{Event selection}

Due to their small extent in frequency and short durations, radio spike bursts are generally difficult to find in spectral radio data. Consequently, the study of solar radio spike bursts, especially in regard to multi-wavelength studies, has been impeded by the sparsity of clearly identified events.

In this work we used notes from routine, near real-time inspections of the AIP radio spectrograms from 1993 August 01 - 2001 December 31 which identified radio spike burst candidates. A systematic search in the spectral data would probably reveal more spike burst events. Since we also require radio imaging information for the spikes we only retained events which overlapped the discrete frequencies of the NRH when 2-D observations were taken (1996 July $01-$ ) and which occurred from 09-15 UT. This reduces the set of candidates considerably, as does requiring overlapping multi-wavelength observations from SOHO EIT (1996 January 01 -) and Yohkoh SXT and HXT (1991 October 01 - 2001 December 14). With this procedure we have found one reasonably well-observed event which is the subject of this paper.

\section{Observations}

\subsection{Whole-Sun observations}

\subsubsection{GOES soft X-ray fluxes}

The radio spike burst event examined here was associated with a solar flaring episode which occurred from 09:36:40-09:41:50 UT on 1997 November 14 at about N18E74 in NOAA Active Region 8108. As viewed from the perspective of the GOES soft X-ray fluxes, this flaring episode was of relatively short duration and appeared superposed on a series of temporally overlapping flaring episodes (see Fig. 1). The sequence of flaring episodes may be due to separate independent flares, or separate, but causally-related, flares, or separate episodes of energy release in a single flare. The relation between these episode will become clearer when we examine other data. In the meantime, for simplicity, we simply refer to the flaring 


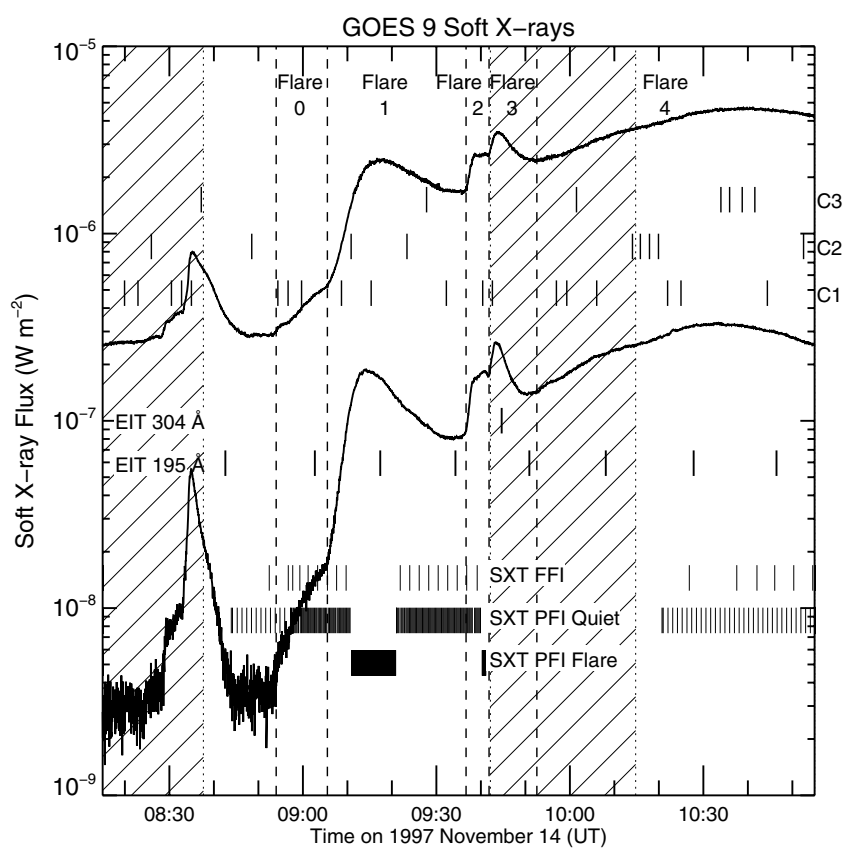

Fig. 2. The soft-ray flux for the low energy channel $(\sim 1-8 \AA$ - the higher flux curve) and the high energy channel ( $\sim 0.5-4 \AA$ - the lower flux curve) from GOES 9 showing the times of SXT full-frame images (FFIs) and partial-frame images (PFIs); EIT 195 and 304 Å images; and LASCO C1, C2, and C3 images. Also shown are the time intervals of the enumerated flares and Yohkoh satellite night.

episodes as "flares", regardless of interpretation. The flares in the sequence had similar peak fluxes $(\mathrm{C} 1-\mathrm{C} 4$ range) but were of significantly differing durations (some several minutes, others several hours). Although the precise intervals of these flares are unclear, we give our best estimates below (based on the GOES soft X-ray fluxes; intervals indicated on Figs. 1 and 2). These flare intervals are not meant to be interpreted too rigidly. Some of the flares may start earlier or end later, or may be part of another flare, or, indeed, all the flares may be part of one flare. Flare 0 occurred from 08:54:00-09:05:30 UT and consisted of a gradual rise in soft X-ray flux prior to Flare 1 . Flare 1 lasted from 09:05:30-09:36:40 UT and was a (relatively) longdecay event (judging from its rate of decrease of flux). Flares 2 and 3 which occurred between $\sim 09: 36: 40-09: 41: 50$ UT and 09:41:50-09:52:35 UT, respectively, were short-duration flares (and, as we will soon see, were associated with radio spike burst emission). Flare 4 was a long-decay event which lasted from $\sim 99: 52: 35$ to at least $\sim 15: 05: 00 \mathrm{UT}$, perhaps as late as $\sim 16: 50$ UT or so. (We also note that a rough back-extrapolation of Flare 4 indicates that Flare 0 may even be the start of Flare 4 (see Fig. 2)). In addition, several flare-like soft X-ray brightenings were observed during the decay phase of Flare 4. These minor flarings will be discussed later in the paper.

The flare of principal concern in this paper is the first radio spike burst-associated flare, Flare 2. However, we also examine the development of the other flares as they provide information which can help us better understand Flare 2 and the radio spike burst emission.

In Fig. 2 we present a zoomed portion of the GOES soft $\mathrm{X}$-ray fluxes near the time of Flare 2 with tick marks indicating the times of some of the imaging data analysed in this paper. Also shown are hatched intervals when Yohkoh was behind the Earth and did not observe the Sun. We will examine and discuss the imaging data in Sect. 3.2.

\subsubsection{AIP radio spectrograms}

The AIP Solar Radio Spectrometers observed the Sun throughout the duration of Flares $0-4$. We now briefly present and describe the principal features observed in the AIP radio spectrograms for each enumerated flare (see also Table 1).

During the time interval of Flare 0 we note an enhancement of pre-existing noise storm (NS) type I bursts and continuum emission centred at $140 \mathrm{MHz}$. The NS was reported during the whole AIP Radio Spectrograph observing day between 100$350 \mathrm{MHz}$. This is independently confirmed by NRH $164.0 \mathrm{MHz}$ reports (Solar Geophysical Data, 1998, 641(I), 112).

In the spectrograms Flare 1 started with a group of type III bursts between 80 and $40 \mathrm{MHz}$ from 09:07:30-09:14 UT (Fig. 3). During the interval of Flare 1 the first decimetric features were seen as faint positively and negatively drifting features between 200 and $>400 \mathrm{MHz}$ at about 09:10 UT. Decimetric emission in the form of broadband pulsations were also observed after 09:22 UT. As we will see later the SXT data indicate that Flare 1 was associated with expanding or ejecting soft X-ray loops. The observed radio features for Flare 1 may be consistent with this interpretation. There was also ongoing NS activity between 100-170 MHz.

For Flares 2 and 3 the AIP spectrograms show radio spike bursts and superposed continuum emission in the frequency range $\sim 200-800 \mathrm{MHz}$ during the time interval $\sim 09: 36: 00-$ 09:52:00 UT. The spectrogram in Fig. 4 reveals isolated radio spike burst emission without simultaneous type III bursts at lower frequencies. In Fig. 5 we present a detailed view of the radio spikes associated with Flare 2. This indicates that three clusters of spike bursts were observed during the interval of Flare 2, 09:36:00-09:39:00UT. We note that strong radio spike bursts are sometimes ordered in chains - similar to noise storm chains (Elgarøy 1977). Figure 5 also indicates that continuum emission occurred during the third cluster of spikes associated with Flare 2. After the spike clusters associated with Flare 2 a group of strong type III bursts was observed below $200 \mathrm{MHz}$ ( 09:42 UT). Spuriously repeated spike bursts may also occur later during the interval of Flare 3 below $300 \mathrm{MHz}$. However, they are not clustered and so are difficult to distinguish from ongoing NS activity in the spectral data. There are also superposed faint type III bursts. We do not have overlapping SXT or HXT data for Flare 3, and, consequently, we exclude this interval from detailed consideration and concentrate on the strong radio spike clusters associated with Flare 2.

Figure 6 shows that the Long Decay Event (LDE) Flare 4 was associated with slowly drifting continuum (onset $\sim 400 \mathrm{MHz}$ at $\sim 09: 53$ UT, leaving our observing range at $\sim 10: 30 \mathrm{UT})$. Aurass et al. (1999) suggested that such slowly drifting faint radio continua may be a radio signature of CMEs. Bastian et al. (2001) using radio imaging data from NRH showed that radio emission similar to that observed for Flare 4 was associated with the moving body of a CME. They classified the radio emission as gyrosynchrotron emission of electrons trapped in an expanding magnetic structure. In our opinion the radio spectral information suggests there were expanding loops and/or a CME associated with Flare 4. As we will see later, this is confirmed from soft $\mathrm{X}$-ray, EUV and white-light coronagraph imaging data.

After the decay of the continuum emission there was ongoing NS emission below $170 \mathrm{MHz}$, as well as isolated continuum patches at higher frequencies. There were also isolated radio bursts after the GOES soft X-ray flux maximum for Flare 4. Some of these will be discussed briefly later in the paper. 
Table 1. Radio spectral data during Flares 0-4 on 1997 November 14.

\begin{tabular}{llcl}
\hline \hline Flare No. & Time (UT) & Frequency (MHz) & Diagnosis \\
\hline 0 & $08: 52-09: 05$ & $100-170$ & NS enhancement \\
1 & $09: 05-09: 37$ & $40-400$ & NS, some faint III, dm features, later dm pulsations \\
2,3 & $09: 37-09: 52$ & $40-800$ & NS, continuum + spikes, some strong III \\
4 & $09: 52-10: 40 \mathrm{U}$ & $40-800$ & NS, drifting continuum \\
\hline
\end{tabular}

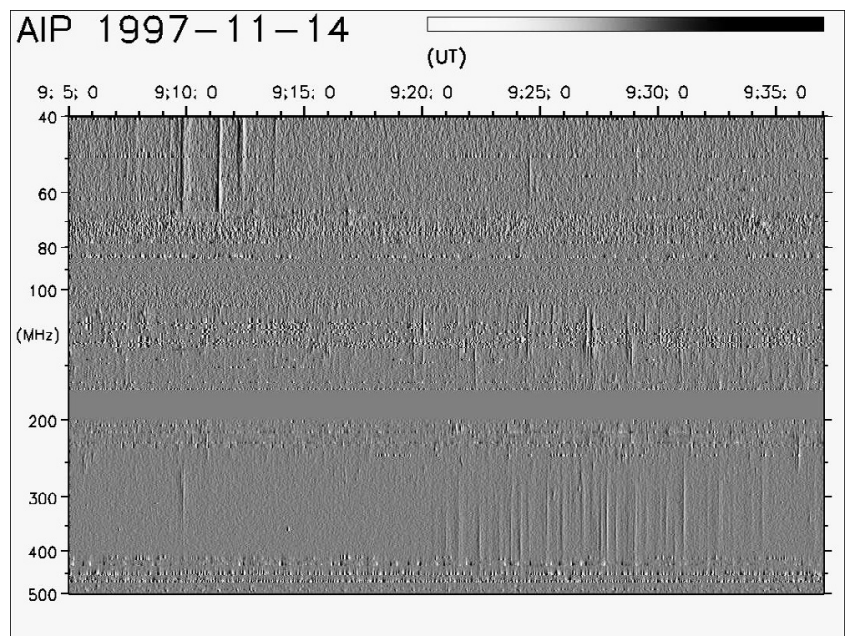

Fig. 3. An AIP spectrogram showing radio emission associated with Flare 1 in the form of the time derivative of the spectrum (to enhance faint features). There was a group of type III bursts between 80 and $40 \mathrm{MHz}$ (09:07.30-09:14 UT). The first decimetric features occurred between 200 and $>400 \mathrm{MHz}$ as faint positively and negatively drifting features at about 09:10 UT, and as broadband pulsations after 09:22 UT. There was ongoing NS activity, enhanced with the pulsations, between 100 and $170 \mathrm{MHz}$.

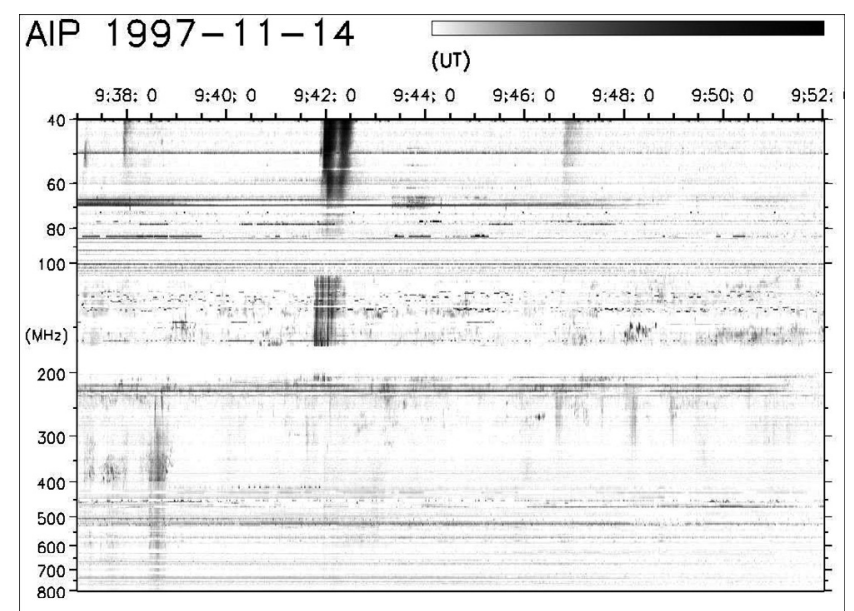

Fig. 4. An AIP spectrogram showing radio emission associated with Flares 2 and 3. The dominant feature is a group of intense type III bursts around 09:42 UT. In this paper we focus on the three continuum enhancements with embedded spike bursts between $200-800 \mathrm{MHz}$ from 09:37-09:39 UT. Spuriously repeated spikes occurred later, too. There were ongoing NS features between 100-170 MHz.

\subsubsection{Hard X-ray and radio fluxes}

In Fig. 7 we show the HXT L channel $(\sim 14-23 \mathrm{keV})$ flux and BATSE DISCLA channel $1(\sim 25-50 \mathrm{keV})$ and channel 2 ( $\sim 50-100 \mathrm{keV})$ fluxes for Flare 2. Moreover, the BATSE data

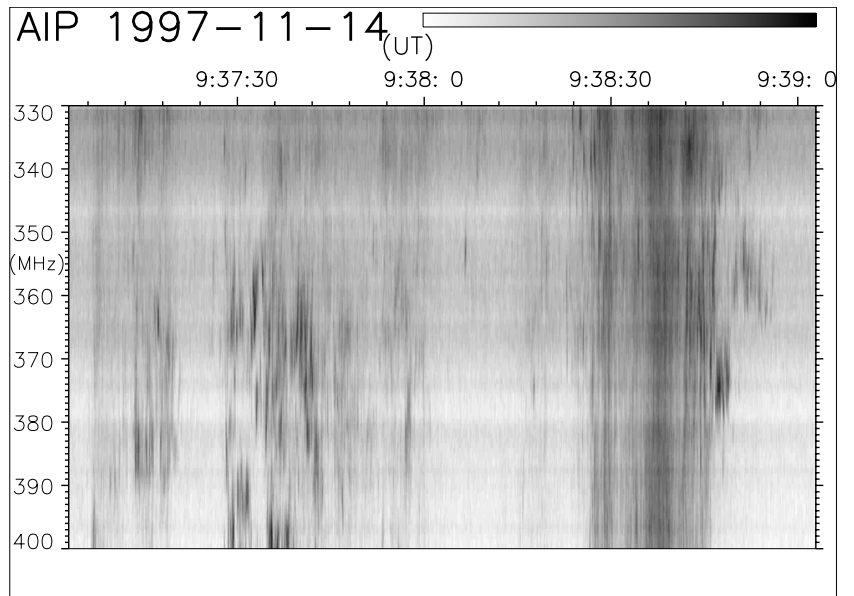

Fig. 5. An AIP spectrogram, enlarged from Fig. 4, showing the spike clouds superposed on continuum emission. The radio emission in this range, 332-400 MHz, was averaged to obtain the radio flux plot shown in Fig. 7.

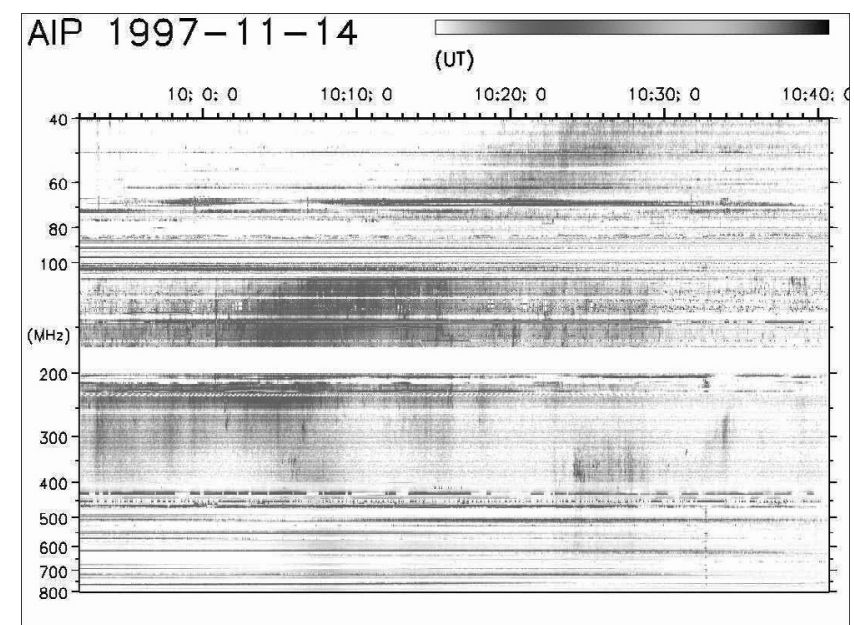

Fig. 6. An AIP spectrogram showing a slowly-drifting radio continuum burst associated with Flare 4.

indicate that the first two clusters of spikes were associated with hard X-rays of energies up to at least $\sim 25-50 \mathrm{keV}$, while the third cluster was associated with hard X-rays of energies up to at least $\sim 50-100 \mathrm{keV}$. Although the HXT L channel is in the energy range where thermal and non-thermal contributions to the hard X-ray flux may occur, the detection of hard X-rays with the BATSE DISCLA detectors clearly indicates that the emission observed by the HXT L channel is, in this case, due to nonthermal energetic electrons.

In the top panel in Fig. 7 we present the frequencyintegrated (from 332-400 MHz, compare Fig. 5) radio flux from the AIP Solar Radio Spectrometers and the HXT L channel 


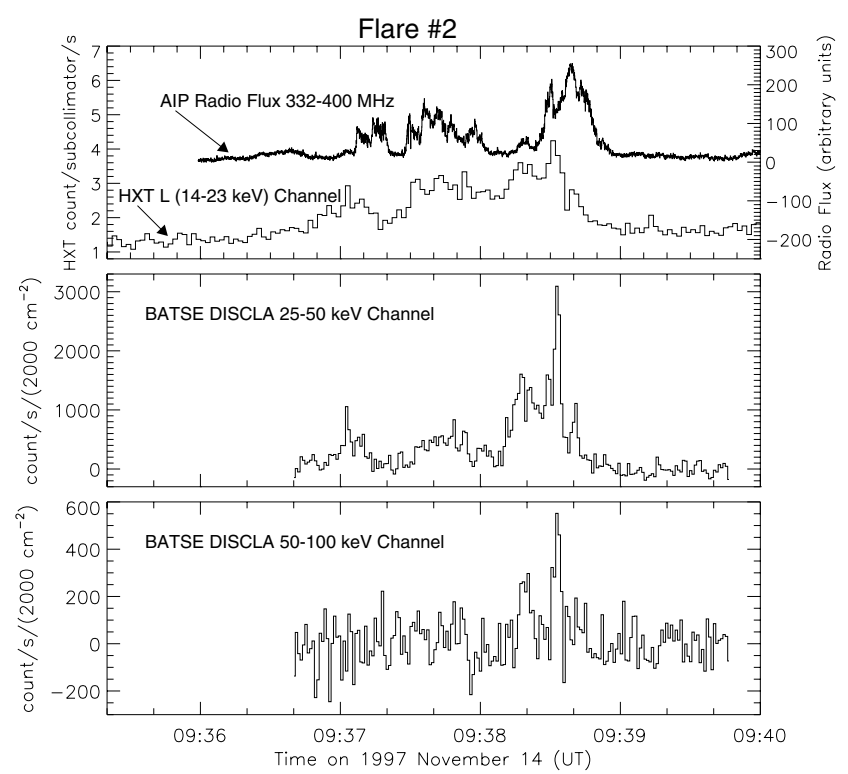

Fig. 7. Hard X-ray fluxes as observed by the HXT L channel $(\sim 14-23 \mathrm{keV})$ and BATSE DISCLA channel $1(\sim 25-50 \mathrm{keV})$ and channel $2(\sim 50-100 \mathrm{keV})$ for the radio spike burst-associated flare, Flare 2. Also shown in the top panel is the frequency-integrated AIP radio flux for the spike bursts associated with Flare 2.

( 14-23 keV) flux. Even judging by eye, these flux plots appear to be similar in general form.

\subsection{Imaging data}

\subsubsection{Yohkoh SXT images}

We now consider the evolution of soft X-ray structures associated with each of the enumerated flares as observed by the SXT.

An overview of the development of the flares as seen in the SXT PFIs is shown in Fig. 8. Flare 0 may be associated with a relatively faint brightening of some loops in the active region. Initially Flare 1 appeared to consist of a loop (which could also be an arcade of loops) with bright footpoints. In later observations Flare 1 appeared to consist of an expanding bright arcade of loops. Flare 2 was associated with one or more small flaring loops located at the leading edge of the active region, remote from the loops observed in Flare 1. Flare 3 was not observed by the SXT. Flare 4 was associated with a bright, expanding arcade of loops which appears to have developed out of magnetic structures stacked in the region of the arcade associated with Flare 1.

Figure 9 shows the early development of Flare 1 in greater detail. The SXT data clearly show an expanding and ejecting faint loop structure above the bright arcade of loops (where the bulk of the soft X-ray emission associated with Flare 1 originates). The soft X-ray ejection is first seen in the SXT images at $\sim 09: 03: 42$ UT. (We conclude that this is a better estimate for the inferred start time for Flare 1 than that based on the GOES data, where the initially low flux for Flare 1 may have masked that of Flare 0.)

When we consider the SXT FFIs, we observe some of the features already noted with the SXT PFIs, but the FFIs reveal another important phenomenon. As the faint soft X-ray feature related to Flare 1 is being ejected a compression of soft X-ray emitting magnetic field structures located at the southern flanks of the ejecting region is observed. We illustrate this compression in Fig. 10 which shows corrected raw images as well as
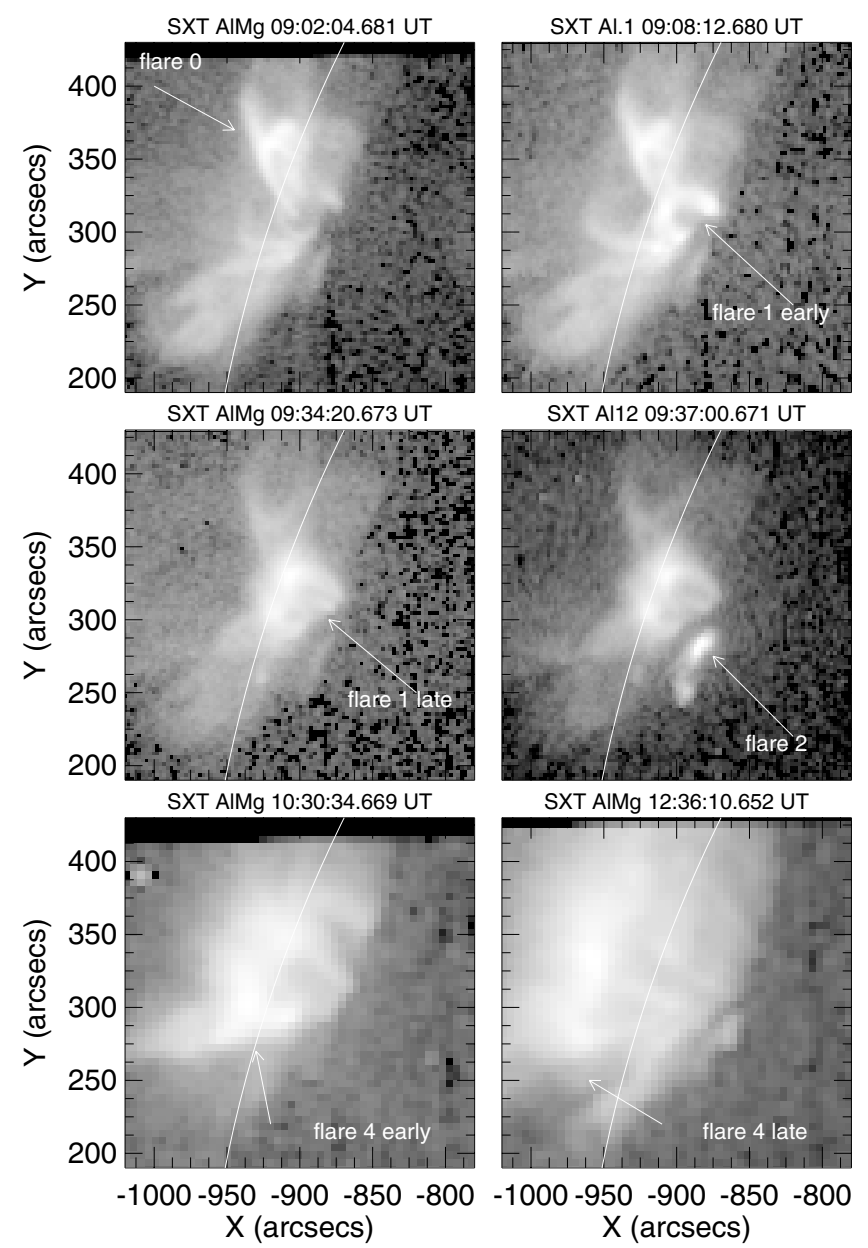

Fig. 8. Portions of corrected SXT partial-frame images (PFIs) showing an overview of the development of the sequence of flares on 1997 November 14. The images have been scaled to show the brightest features in each image (i.e., relative differences between images are not retained). Note Flare 3 was not observed by SXT but SOHO EIT data show it originated from the same region as Flare 2.

difference images. The portions of the FFIs' in this figure show the soft X-ray ejection associated with Flare 1 as well as compressed magnetic field structures, which are most apparent as the white and black stripe pattern in the difference images. Reexamination shows that this compression is also seen (faintly) in the SXT PFIs. The compressed magnetic field structures appear to be highly inclined coronal loops at the periphery of the active region. The compression of magnetic structures is observed even more clearly in the EIT images which we will show and discuss below.

\subsubsection{SOHO EIT images}

The time cadence of EIT images is generally no better than one whole-Sun image every $\sim 16 \mathrm{~min}$ or so. Consequently, we do not observe the development of some of the flares as well as with the SXT. However, other features are seen more clearly.

Although we do not present an image of it in this paper, we note that Flare 3 (as observed in the EIT data) appeared to originate from the same or similar set of magnetic field structures located in the same region as Flare 2 (as observed in the SXT data). 

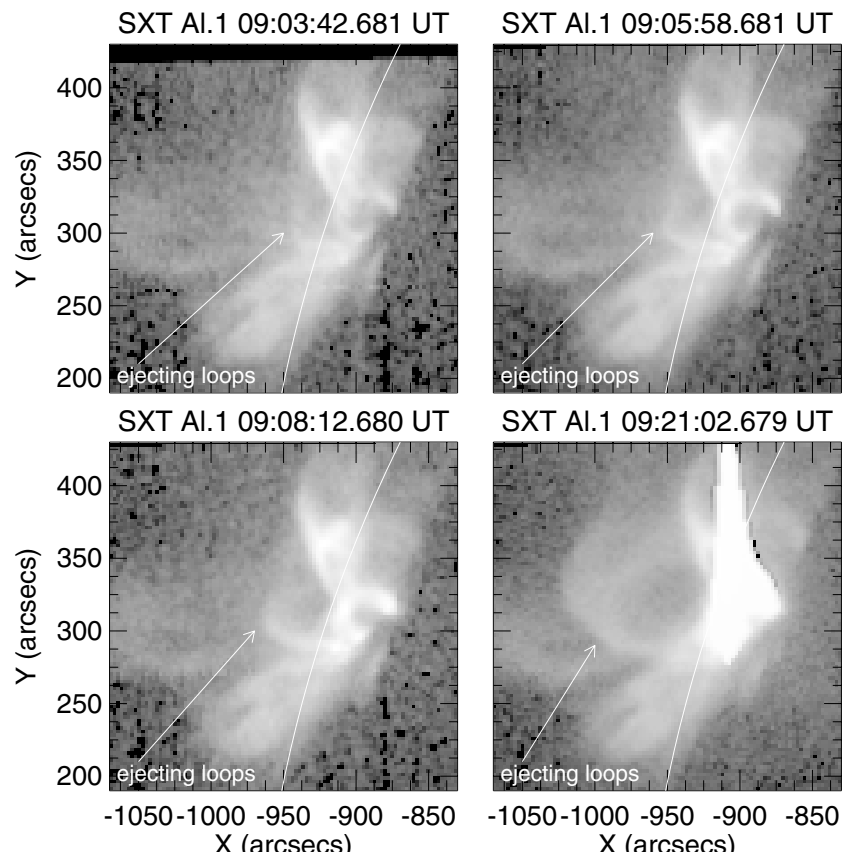

SXT Al.1 09.21:02.679 UT

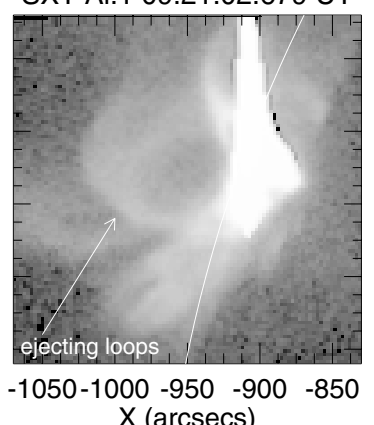

Fig. 9. Portions of corrected SXT partial-frame images (PFIs) showing faint soft X-ray emitting features which appear to be in the form of ejecting and expanding loops associated with Flare 1.
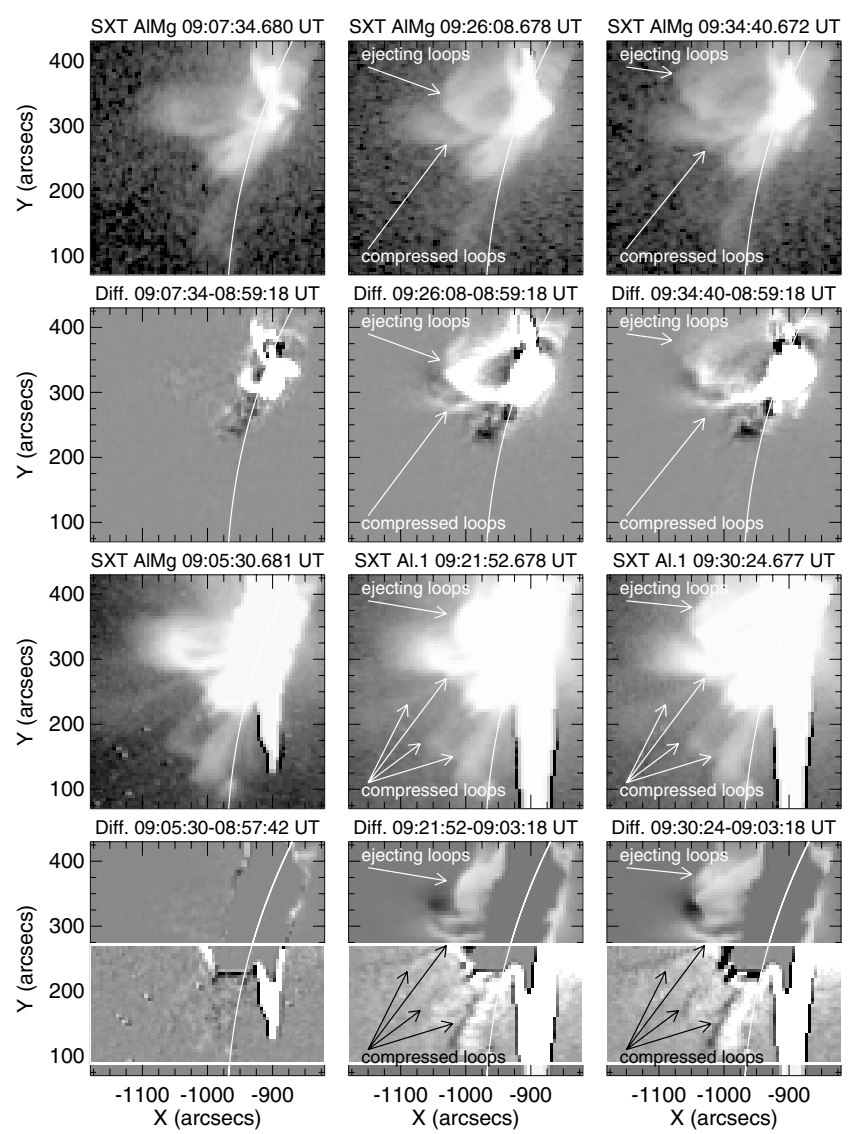

Fig. 10. Portions of SXT full-frame images (FFIs). Rows 1 and 3 show corrected raw data, while rows 2 and 4 show difference images. For the lower portion of row 4, we superposed difference images truncated over a narrower data number (DN) difference range (absolute value 20 as opposed to 400). The images show the expanding and ejecting soft $\mathrm{X}$-ray loops and the compression of soft X-ray emitting magnetic field structures.
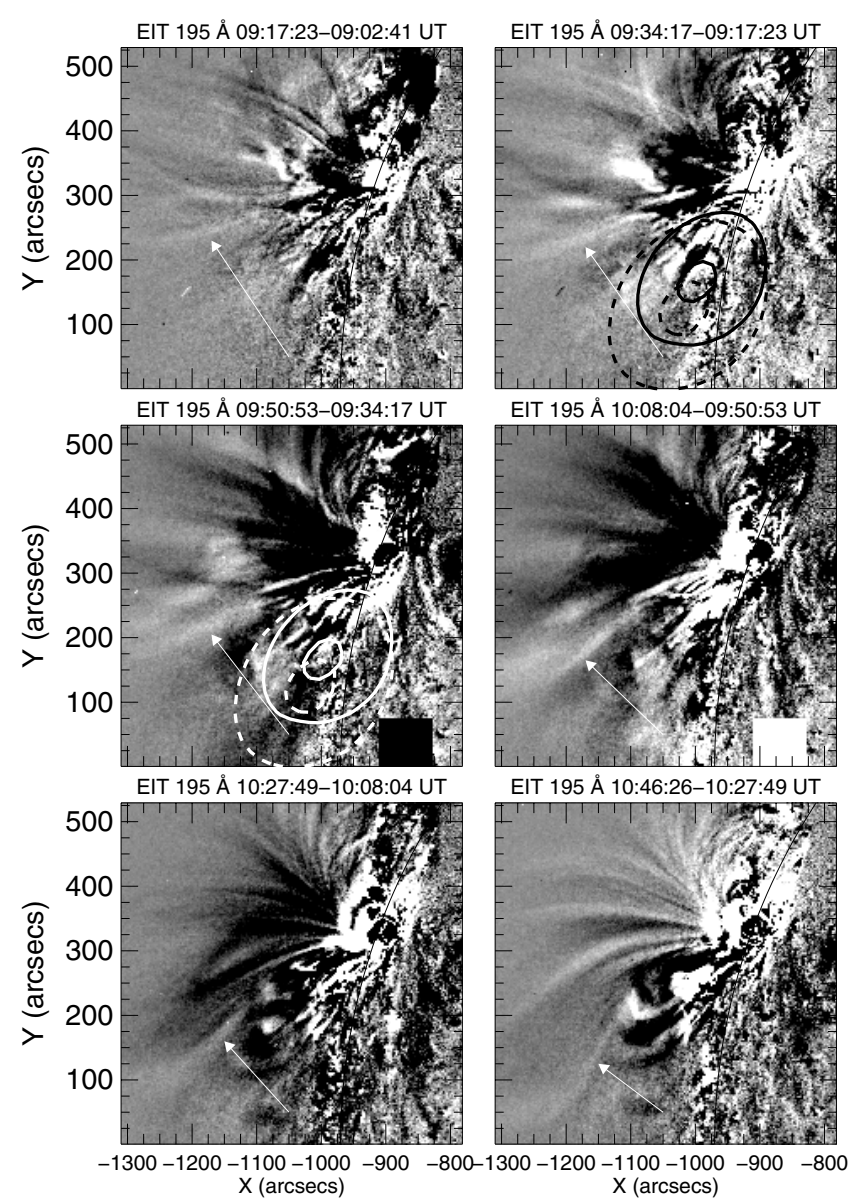

Fig. 11. Portions of running difference EIT images showing largescale compression of magnetic field structures (indicated by arrows). Dimming of the EIT images in the vicinity of the soft X-ray ejection region seen in Fig. 9 is also apparent. Also show on two of the panels are the $60 \%$ and $90 \%$ contour levels for the NRH $327.0 \mathrm{MHz}$ source (dashed lines) and $410.5 \mathrm{MHz}$ source (solid lines) of the radio spike burst at 09:38:30 UT.

The most striking feature revealed by the EIT data is the clear compression of the magnetic structures. As already noted, the SXT data show expanding and ejecting loops associated with Flare 1. However no ejection is seen in the SXT data for Flare 4. This may be due to the gap in the temporal coverage for Flare 4. The SXT data show an expanding arcade for Flare 4, which could be taken as a proxy for an earlier mass ejection. The EIT data do not show the leading edge of the mass ejection associated with Flare 1 nor one for Flare 4. This may be because the ejecting material was at too high a temperature to be observed by EIT. Instead a general dimming due to evacuation in the vicinity of the faint soft X-ray ejecting loop region is seen in the EIT data. In addition, apparent motion of EUV emitting plasma is observed at the flanks of the faint soft X-ray ejecting loop region. This motion is illustrated in Fig. 11 which shows a sequence of EIT difference images. Also shown in Fig. 11 are contours for the radio spike burst at 09:38:30 UT as imaged by the NRH. As the spikes occur from near 09:37-09:40 UT, neither of the two EIT (difference) images overlap the spike interval. The 09:34-09:17 UT image shows features before the spikes, while the 09:50-09:34 UT image shows features after the spikes. These illustrate that the motion of the EUV emitting plasma is in the general direction of the radio spike burst sources. Moreover, the motion is observed to occur from the time of Flare 0 through 

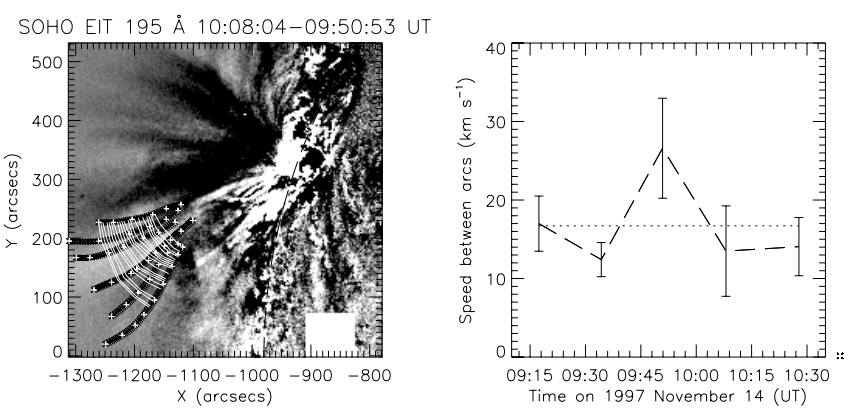

Fig. 12. The left-hand panel shows a difference image from Fig. 11 with superposed white + marks indicating points clicked along the arcs, interpolated points between them (black x [which are not resolved in the figure and appear as thick black lines]), and the subset of shortest distance line segments (white lines) used to estimate the speed between arcs. The right-hand panel shows the mean of the speeds between successive arcs estimated from the line segments, with the error bars indicating the standard deviation of the values. The overall average speed is indicated by the dotted line.

to the time of Flare 4. Bearing in mind that mass ejection signatures are suggested in the SXT and spectral radio data for Flares 1 and 4, it is therefore reasonable to associate the EUV compression and dimming with one or both of these mass ejections. We note that the compressing EIT (as well as the compressing SXT) features appeared to bounce back (i.e., restore to close to their previous position) leading us to associate these features with compressing loops rather than with ejecta.

The higher quality of the EIT data with regard to the compression means that we can trace portions of arcs of the moving and apparently compressing magnetic structures to estimate the speed of compression. The technique we used is, essentially, a ray-tracing technique similar to Huygens' principle which is used to predict the propagation of a wave-front. Hudson et al. (2003) used this technique to examine propagating wave-like disturbances seen in SXT images. First we visually selected points along the bright leading edges of the compressing loops (which were seen at the inferred flank of the mass ejection) by "pointing and clicking". Then we spline interpolated between these points to generate a larger number of points along each arc. Next we determined the closest spline-interpolated points between successive arcs. Determining the distance between each pair of points and knowing the times of the arcs (images) we infer the instantaneous speeds at different points along the arc. From the moment of these speeds we determine the average speed between successive arcs and its standard deviation. The average of the average speeds between successive arcs then gives us the overall average speed of compression. With this technique we find that the overall average speed of compression was $17 \pm 7 \mathrm{~km} \mathrm{~s}^{-1}$. Figure 12 shows the locations of some of the estimated arcs superposed on a single image as well as the average speeds between successive arcs and the overall average speed.

\subsubsection{Yohkoh HXT images}

The HXT only observed hard X-rays from Flare 1 and Flare 2. We are able to make a few images of Flare 1 with the HXT L channel ( 14-23 keV) data. They show hard X-ray emission from the footpoints of the arcade of loops as well as from the loop-top region. These are not shown here.

For Flare 2 the HXT observations were in quiet-mode throughout the duration of the flare and thus we only have hard $\mathrm{X}$-rays in the L channel $(\sim 14-23 \mathrm{keV})$. Due to the relatively low

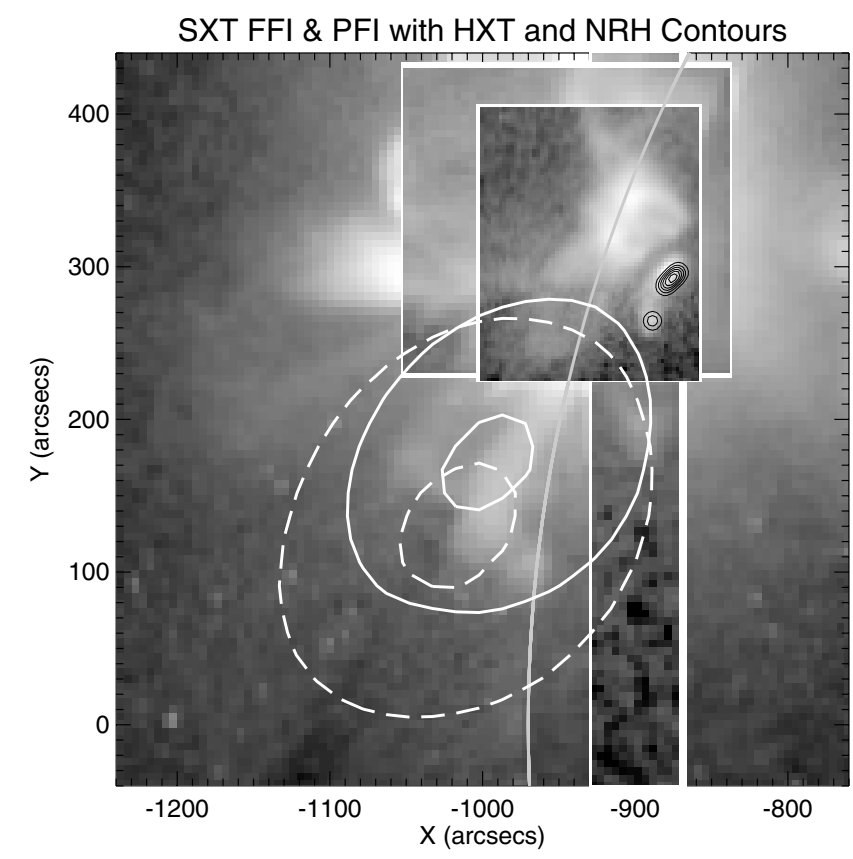

Fig. 13. An SXT PFI taken at 09:37:00 UT showing the bright arcade of Flare 1 and the small flaring structures of Flare 2, superposed on a portion of an FFI taken at 09:32:32 UT (with saturated portions covered with an FFI taken at 09:39:10 UT). Shown in black contours are the HXT L channel $(\sim 14-23 \mathrm{keV})$ sources constructed by accumulating counts over the interval 09:36:45-09:38:50 UT. The dashed white contours show the $60 \%$ and $90 \%$ contour levels for the NRH $327.0 \mathrm{MHz}$ source of the radio spike burst at 09:38:30 UT. Similarly the solid white contours show the NRH $410.5 \mathrm{MHz}$ source of the radio spike burst at 09:38:30 UT.

intensity and poor time cadence we are only able to make a single image for Flare 2 by accumulating the counts over the entire duration of that flare. Figure 13 shows an SXT image with superposed contours (in black lines) of an HXT image constructed using the pixon algorithm (Metcalf et al. 1996) for Flare 2. The hard X-ray emission consists of a comparatively extended and intense hard X-ray source to the north (which may be due to a loop source or two unresolved footpoint sources) which lies at the location of the main flaring loop structure observed in the SXT images for Flare 2. To the south is a fainter hard X-ray source associated with a fainter soft X-ray structure related to Flare 2.

\subsubsection{Nançay radioheliograph data}

The NRH observed the radio spike bursts at the frequencies $327.0 \mathrm{MHz}$ and $410.5 \mathrm{MHz}$. We find no significant motion of the NRH sources during the interval of spike burst emission at both frequencies.

In Fig. 13 we present a composite image, showing SXT, HXT and NRH features. The largest portion of the grayscale image is an SXT FFI taken at 09:32:32 UT, superposed on this is another FFI (to replace saturated portions) and a PFI. The PFI portion was taken at 09:37:00 UT and shows the bright arcade of Flare 1 as well as the small flaring structures associated with Flare 2. Superposed on that are black contours indicating the hard X-ray sources associated with Flare 2. Shown in white contours are the $60 \%$ and $90 \%$ contour levels for the NRH sources during the spike burst at $327.0 \mathrm{MHz}$ (dashed lines) and 410.5 MHz (solid lines) near the time of the PFI of Flare 2. 
From this figure it is clear that the radio spike burst emission was remote from the hard and soft X-ray sources associated with Flare 2. We find that the centroid of the site of main emission in hard X-rays was $\sim 122 \mathrm{Mm}$ and $\sim 154 \mathrm{Mm}$ (as seen in projection) from the centroids of the radio spike emission seen at $410.5 \mathrm{MHz}$ and $327.0 \mathrm{MHz}$, respectively. Furthermore, the spike bursts were observed in the vicinity of the compression of the magnetic structures as seen in the SXT and EIT data. Note the compression of these structures was observed to start before Flare 2 and to continue well into the decay phase of Flare 4.

Note that a CME was observed in the SOHO Large Angle Spectroscopic Coronagraph (LASCO) (Brueckner et al. 1995) during and immediately after the sequence Flares $0-4$. This, and the above results, lead us to infer the following sequence of events as a possible scenario giving rise to the spike burst emission. Flare 1, associated with expanding and ejecting soft X-ray loops or, possibly, (an early stage of) Flare 4 (which showed inferred ejective signatures in the spectral radio data - see the drifting continuum onset in Fig. 6 and Aurass et al. 1999 and later in soft X-ray images) may have been associated with the CME. During the course of Flare 1 or Flare 4 the associated mass ejection compresses magnetic structures at its flank giving rise to magnetic reconnection, particle acceleration and radio spike burst emission in the vicinity of the compression. Some of the accelerated particles reach lower regions of the solar atmosphere giving rise to hard X-ray emission and ultimately Flare 2.

\section{After Flare 2}

The decay phase of Flare 4 appears to show several subsidiary flare-like features in the GOES soft X-ray data. In the top part of Fig. 14 we show the GOES soft X-ray fluxes for the decay phase of Flare 4 and enumerate these features which we refer to here as soft X-ray blips. Soft X-ray blips 7-10 might perhaps be considered to be small flares rather than flare-like brightenings.

We also examined the SXT and EIT data to identify the locations associated with these brightenings. To illustrate our finding we present observations of the blips seen by SXT in the bottom part of Fig. 14. Summarising, the SXT and EIT images reveal that soft X-ray blips 3 and 6 were apparently associated with magnetic field structures near Flare 1, while soft X-ray blips 5, 8,9 , and 10 show features from the same region as Flare 2. Soft X-ray blip 4 shows emission from both sites. We do not know the location of soft X-ray blips 0, 1, 2, and 7. However it appears reasonable to assume that the emission might have occurred in the same general vicinity as the two soft X-ray blip emission sites as typified by soft X-ray blips 3 and 5 .

We examined the AIP radio spectrograms to identify any radio features associated with the soft X-ray blips. Most do not show obvious radio features. However, soft X-ray blips 2 and 4 are found to have radio spike burst emission. Only soft X-ray blip 4 which has radio spikes bursts from $\sim 210-290 \mathrm{MHz}$ over the interval 13:49:35-13:49:53 UT overlaps one of the frequencies of the NRH (i.e., 236.6 MHz). We find that NRH spike burst source to be located at roughly $(-870,60)$ in arc seconds from disk centre. The location of these spikes are not inconsistent with the previous location of the earlier spikes, if we make allowance for solar rotation of an elevated source region.

The SXT and EIT $195 \AA$ data show that the compression of the magnetic field starts during Flare 1 and continues until after the peak in the soft X-ray flux for Flare 4. Specifically, the EIT data indicate clear compression from about 09:02-10:27 UT while, images at 10:46 UT and 11:02 UT show the "bouncing back" of the loops. After 10:27 UT a bright flaring arcade is seen

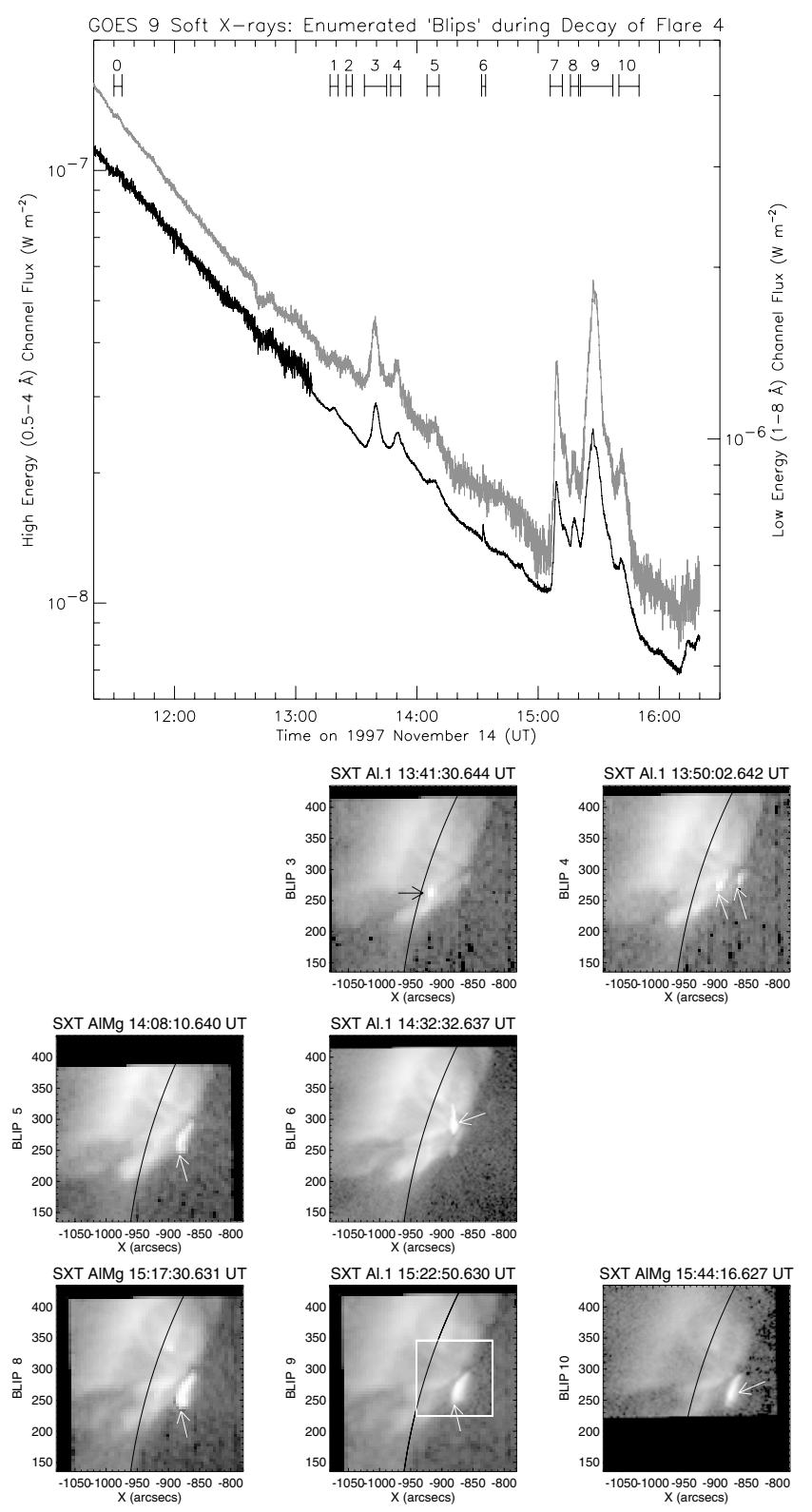

Fig. 14. The graph at the top shows the GOES soft X-ray fluxes for the decay phase of Flare 4, identifying and enumerating subsidiary flarelike brightenings, which we refer to as soft X-ray blips. The bottom part of the figure shows SXT images of some of the soft X-ray blips. In each image we replaced the ordinate label with a label identifying the enumerated soft X-ray blip (as given in the graph).

(in the EIT data) to grow underneath the large scale loops which earlier showed apparent "compressing and bouncing" motions. Could the expanding arcade also have compressed loops peripheral to the active region? This is not clear from the EIT data. However, the SXT (half resolution Al.1) PFIs appear to show compression from 12:00-14:15 UT. As these images observed a relatively small field of view, as well as features above the limb, it is difficult to be as confident about the compression as for the earlier times. Since we find radio spikes associated with Flares 2 and 3, and some of the flare-like brightenings during the decay of Flare 4 appear to originate near the location of Flare 2 and have radio spikes, we suggest that continued compression of magnetic field structures may have led to repeated radio spike burst emission and flaring. 


\section{Discussion and conclusions}

Earlier work by Krucker et al. (1997) and Paesold et al. (2001) presented images of radio spike burst sources in relation to soft X-ray emission, while Benz et al. (2002) showed the first reported example of images of radio spike bursts in relation to hard $\mathrm{X}$-ray sources. These earlier works provided essentially snapshots of contextual information associated with the radio spike burst emission. It was not possible to infer the macroscopic dynamical processes associated with the formation of the spike bursts. Consequently, the relation between the radio spike bursts and the soft and hard X-ray sources and the overall development of the flare was unclear. However, one result noted at the time was that the radio spike bursts were located remote from the energy deposition site as indicated by the X-ray emission signatures. This, and the fact that the radio spike bursts occurred during the decay phase of the flares, led Benz et al. (2002) to suggest a paradigm shift and conclude that radio spike burst sources were questionable as tracers of the main flare particle acceleration site.

In this paper we also provide an example of radio images of solar radio spike bursts in relation to associated simultaneous hard X-ray emission. Similar to the work of Benz et al. (2002) we find the spike bursts to be remote from the hard X-ray sources. In addition, in this paper, since our event was a nearlimb event, we are able to show, for the first time, the location of solar radio spike bursts in relation to the dynamics of coronal structures associated with flaring and CME development as observed in soft X-rays and in EUV.

In this paper we present the results of an analysis of multiwavelength data for a solar radio spike burst event which was observed on 1997 November 14 in spectrograms taken by the AIP Solar Radio Spectrometers. In terms of the temporal context we find that the spike burst event was itself associated with a short-duration flare which appeared (temporally) superposed on two gradual flares. Spectral radio data show that the gradual (or LDE) flare, Flare 4, was associated with faint drifting radio continuum, which has been linked with coronal mass ejections in the past (Aurass et al. 1999). Soft X-ray imaging data from the SXT reveal the ejection of a coronal loop structure during the LDE flare, Flare 1. The expanding bright arcade of Flare 4 may also be taken as an indirect signature for mass ejection. Dimming/evacuation was also observed in EIT images in the vicinity of the SXT ejection during the sequence of flares, Flares 0-4. LASCO data show a coronal mass ejection associated with the sequence of flares supporting the inferences from the low-corona SXT and EIT images and the spectral radio observations. Furthermore, the mass ejection appeared to be associated with the compression of magnetic field structures at an inferred flank of the CME (here observed at the southern periphery of the flaring active region) as observed in SXT and EIT data.

Using radio imaging data from the Nançay Radioheliograph we are able to determine the location of the spike burst emission sites in relation to the hard X-ray sources as well as the relation with the dynamical development of the coronal environment as seen in soft X-rays and EUV. We find that the spike burst emission centroid (at $410.5 \mathrm{MHz}$ ) originated high in the corona, $\sim 122 \mathrm{Mm}$ above the soft X-ray and hard X-ray sources of the associated flare. Moreover, radio images of the spike bursts show that they were temporally and spatially associated with the compression of the magnetic field structures due to a flare-associated CME. We estimate the speed of compression from the EIT images to be $\sim 17 \pm 7 \mathrm{~km} \mathrm{~s}^{-1}$.
Theories of fast magnetic reconnection (Priest \& Forbes 2000 ) predict an inflow speed of $\sim 0.1-0.01 \times$ the Alfvén speed, $v_{\mathrm{A}}$. If we assume a coronal magnetic field, $B \approx 1-10 \mathrm{G}$ and an electron number density, $n \approx 10^{9} \mathrm{~cm}^{-3}$ (corresponding to $300 \mathrm{MHz}$ ) at the reconnection site, this implies $v_{\mathrm{A}} \approx 69-690 \mathrm{~km} \mathrm{~s}^{-1}$ at the reconnection site. With these assumptions the inflow speeds for magnetic reconnection should be in the range $\approx 0.7-70 \mathrm{~km} \mathrm{~s}^{-1}$. Thus the average speed we estimate for the compression seen in the EIT images appears to be within the range of inflow speeds expected from theories of driven magnetic reconnection. It is also similar to the speed of reconnection inflow inferred from other observations (Yokoyama et al. 2001; although cf. Chen et al. 2004).

For the specific event analysed in this paper we find that the spike burst emission is clearly associated with secondary flare energy release induced by an earlier flare-associated CME. These observations thus provide evidence for a new category of sympathetic flaring not discussed in detail previously, where a large flare-associated coronal mass ejection compresses adjacent magnetic structures leading to nearby, but remote, small-scale flaring.

Examination of spectral radio data, as well as SXT and EIT data, for times after the main spike burst event examined in this paper, indicate that the expansion of the CME associated with the earlier sequence of flares led to continued compression of the magnetic structures at its flanks, repeated flarings in the same general vicinity as that associated with the first radio spike burst, and, in some cases, additional examples of radio spike burst emission.

Although detailed comparisons of the locations and development of the various radio emissions observed during the course of Flares $0-4$ are beyond the scope of this paper, we did briefly examine the location and development of the NS emission. We determined the location of the NS emission (at $164.0 \mathrm{MHz}$ ) in relation to the NRH imaging data shown here. The NS source consists of sub-sources of varying intensity, but the source centroids were all $\geq 350^{\prime \prime}$ north of Sun centre and between 760-1045" east of Sun centre. Thus the NS emission was located north of our spike burst sources. Consequently, the differing locations lead us to conclude that, for this event, the NS and the radio spike burst emissions are unrelated to each other.

Summarising, our results lead us to propose the following scenario: A CME associated with an earlier flare within the active region led to the compression of coronal magnetic structures at the periphery of the active region. This compression probably led to magnetic reconnection, which in turn led to particle acceleration and radio spike burst emission in the vicinity of the energy release site. Some accelerated particles travelled towards the lower corona giving rise to, ultimately, the hard and soft X-ray flare signatures. The continued expansion of the coronal mass ejection led to repeated magnetic reconnection, particle acceleration, radio spike burst emission signatures and flaring.

If we identify the main flare energy release with Flare 1 or Flare 4 then we concur with Benz et al. (2002) that the radio spike burst emission was not directly associated with the main flare electron acceleration. Since the spike burst emission occurred in the course of a comparatively minor flare energy release induced by the expansion and compression caused by a (flare-associated) CME, we associate it with secondary electron acceleration. Nonetheless, we do conclude that the spikes were associated with driven magnetic reconnection during flare energy release, albeit a relatively minor flare. As is already wellknown, not all solar flares are accompanied by radio spike bursts. What are the additional requirements for such emissions? Based 
on the event examined here, we speculate that spikes indicate secondary electron acceleration induced by the rearrangement of coronal magnetic fields associated with the main flare energy release (such as the CME-induced flaring found here).

Whether solar radio spikes generally occur with the scenario outlined above requires further investigation. We anticipate that studies using the more recent RHESSI and TRACE data, along with NRH and SOHO EIT and LASCO data will provide further insight into the origins of these bursts. Looking further ahead the Frequency-Agile Solar Radiotelescope (FASR) (Bastian 2004) will provide radio observations of hitherto unprecedented quality and resolution. We anticipate FASR will lead to major advances in our understanding of radio spike bursts and similar radio bursts closely associated with the flare energy release process.

Acknowledgements. We thank T. Kosugi and the personnel at the Sagamihara Campus of the Japan Aerospace Exploration Agency (JAXA) for their hospitality during the early stages of this research. The Yohkoh data used here are courtesy of the Institute of Space and Astronautical Science (ISAS) of Japan The Yohkoh mission was a Japanese project prepared and operated by an international team of Japanese, US, and UK scientists under the financial support of ISAS, NASA, and the Particle Physics and Astronomy Research Council (PPARC) of the UK, respectively. We are grateful to K.-L. Klein and A. Kerdraon for generously providing the Nançay Radioheliograph data used in this paper. The Nançay Radio Observatory is funded by the French Ministry of National Education, Higher Education and Research, the Centre National de la Recherche Scientifique (CNRS), and the French Regional Council of Centre. The EIT data used here are courtesy of the SOHO EIT Consortium. SOHO is a project of international cooperation between ESA and NASA. The GOES data are courtesy of the World Data Center A for Solar-Terrestrial Physics, NGDC, NOAA E/GC2, 325 Broadway, Boulder, Colorado 80303, USA. We thank A. Klassen for help in searching for suitable spike events. We also thank M. Karlický, G. Trottet, S. Krucker, L. Fletcher and L. F. A. Teodoro for stimulating discussions and comments. J.I.K. gratefully acknowledges financial support from PPARC. H.A. acknowledges the support of the Japan Society for the Promotion of Science (JSPS) and the Deutscher Akademischer Austauschdienst (DAAD). We thank the referee, N. Vilmer, for comments and suggestions which helped improve this paper.

\section{References}

Aschwanden, M. J., \& Güdel, M. 1992, ApJ, 401, 736

Aschwanden, M. J., Wiehl, H. J., Benz, A. O., \& Kane, S. R. 1985, Sol. Phys., 97, 159

Aurass, H., \& Mann, G. 2004, ApJ, 615, 526
Aurass, H., Vourlidas, A., Andrews, M. D., et al. 1999, ApJ, 511, 451

Bárta, M., \& Karlický, M. 2001, A\&A, 379, 1045

Bastian, T. S. 2004, in Solar and Space Weather Radiophysics: Current Status and Future Developments, ed. D. E. Gary, \& C. O. Keller, (Dordrecht: Kluwer Academic Publishers), Astrophysics and Space Science Library, 314, 47

Bastian, T. S., Pick, M., Kerdraon, A., Maia, D., \& Vourlidas, A. 2001, ApJ, 558, L65

Benz, A. 0., 1986, Sol. Phys., 104, 99

Benz, A. O., Saint-Hilaire, P., \& Vilmer, N. 2002, A\&A, 383, 678

Brueckner, G. E., Howard, R. A., Koomen, M. J., et al. 1995, Sol. Phys., 162, 357

Chen, P. F., Shibata, K., Brooks, D. H., \& Isobe, H. 2004, ApJ, 602, L61

Delaboudinière, J.-P., Artzner, G. E., Brunaud, J., et al. 1995, Sol. Phys., 162, 291

Domingo, V., Fleck, B., \& Poland, A. I. 1995, Sol. Phys., 162, 1

Elgarøy, Ø. 1997, Solar Noise Storms, (Oxford: Pergamon Press)

Fletcher, L., \& Martens, P. C. H. 1998, ApJ, 505, 418

Heyvaerts, J., Kerdraon, A., Mangeney, A., Pick, M., \& Slottje, C. 1978, A\&A, 66,81

Hudson, H. S., Khan, J. I., Lemen, J. R., et al. 2003, Sol. Phys., 212, 121

Kerdraon, A., \& Delouis, J.-M. 1997, in Coronal Physics from Radio and Space Observations, ed. G. Trottet, (Berlin: Springer-Verlag), Lect. Notes in Phys., 483, 192

Kosugi, T., Makishima, K., Murakami, T., et al. 1991, Sol. Phys., 136, 17

Krucker, S., Aschwanden, M. J., Bastian, T. S., \& Benz, A. O. 1995, A\&A, 302, 551

Krucker, S., Benz, A. O., \& Aschwanden, M. J. 1997, A\&A, 317, 569

Mann, G., Aurass, H., Voigt, W., \& Paschke, J. 1992, in Coronal Streamers, Coronal Loops and Coronal and Solar Wind Composition, (Noordwijk: ESA), ESA SP-348, 129

Mann, G., Aurass, H., \& Warmuth, A. 2006, A\&A, 454, 969

Masuda, S., Kosugi, T., Hara, H., Tsuneta, S., \& Ogawara, Y. 1994, Nature, 371, 495

Messmer, P., \& Benz, A. O. 2000, A\&A, 354, 287

Metcalf, T., Hudson, H. S., Kosugi, T., Puetter, R. C., \& Piña, R. K. 1996, ApJ, 466,585

Ogawara, Y., Takano, T., Kato, T., et al. 1991, Sol. Phys., 136, 1

Paesold, G., Benz, A. O., Klein, K.-L., \& Vilmer, N. 2001, A\&A, 371, 333

Priest, E. R., \& Forbes, T. 2000, Magnetic Reconnection: MHD Theory and Applications (Cambridge: Cambridge University Press)

Somov, B. V., \& Kosugi, T. 1997, ApJ, 485, 859

Sui, L., \& Holman, G. D. 2003, ApJ, 596, L251

Tsuneta, S., Acton, L. W., Bruner, M. E., et al. 1991, Sol. Phys., 136, 37

Tsuneta, S., Masuda, S., Kosugi, T., \& Sato, J. 1997, ApJ, 478, 787

Veronig, A. M., \& Brown, J. C. 2004, ApJ, 603, L117

Wheatland, M. S., \& Melrose, D. B. 1995, Sol. Phys., 158, 283

Yokoyama, T., Akita, K., Morimoto, T., Inoue, K., \& Newmark, J. 2001, ApJ, 546, L69 